\title{
Cambio en el patrón de presentación del cáncer de próstata en Oviedo en los últimos 10 años: diagnóstico y tratamiento (Parte II)
}

\author{
Álvarez-Múgica M, Fernández Gómez JM, Escaf Barmadah S, Jalón Monzón A, \\ González Álvarez RC, Regadera Sejas FJ.
}

Servicio de Urología. Hospital Universitario Central de Asturias. Oviedo.

Actas Urol Esp. 2006;30(10):980-986

\section{RESUMEN}

\section{CAMBIO EN EL PATRÓN DE PRESENTACIÓN DEL CÁNCER DE PRÓSTATA EN OVIEDO EN LOS ÚLTIMOS 10 AÑOS: DIAGNÓSTICO Y TRATAMIENTO (PARTE II)}

Objetivos: Describir el patrón de diagnóstico y tratamiento en los pacientes diagnosticados de cáncer de próstata en nuestro servicio, comparando los datos obtenidos en el año 1995 y en el 2004.

Material y métodos: Se revisaron las 216 historias clínicas de los pacientes diagnosticados en 1995 y 2004, recogiéndose unos datos según un protocolo establecido.

Resultados: Se observaron diferencias significativas en las medias del número de cilindros obtenidos en las biopsias en el año $1995^{2,3}$ frente a las del $2004^{1,6}$. Respecto al tratamiento, en el año 1995 predominó la deprivación androgénica seguida de la prostatectomía radical mientras que en el año 2004 la terapia más utilizada fue la radioterapia (externa y/o braquiterapia), seguida por la deprivación androgénica y la prostatectomía radical. Resultaron factores independientes para plantear el tipo de tratamiento la edad $(\mathrm{OR}=5,6)$ y el grupo de riesgo (OR=2,8) en el año 2004 y únicamente la edad ( $>70$ años vs $\leq 70$ años) en el año 1995.

Conclusiones: La evolución experimentada en las distintas técnicas terapéuticas ha convertido al cáncer de próstata en una enfermedad multidisciplinar obteniendo una gran mejoría en los resultados del tratamiento. Pese a todo esto, el desconocimiento existente en la actualidad acerca de la historia natural del cáncer de próstata y de su evolución en la mayoría de los casos, hace que sea necesario la realización de grandes estudios prospectivos, los cuales se están llevando a cabo, para determinar si el cribado del cáncer de próstata mediante el uso del PSA puede ser útil o no.

Palabras claves: Cáncer de próstata. Hormonoterapia. Quimioterapia.

\footnotetext{
ABSTRACT

CHANGES IN THE PRESENTATION PATTERN OF THE PROSTATE CANCER IN OVIEDO IN THE LAST 10 YEARS: DIAGNOSTIC AND TREATMENT (PART II)

Objectives: To describe and compare the diagnostic and treatment factors of prostate adenocarcinomas diagnosed in our department in the years 1995 and 2004.

Material and method: We review the 216 patients diagnosed in both years, recording several features.

Results: Significant differences were found in the mean of cylinders taken in the biopsies, being higher in $2004^{1,6}$ than in $1995^{2,3}$. Hormonal deprivation was the predominant treatment in 1995 followed by radical prostatectomy, while in 2004 radiotherapy (external beam or braquitherapy) was the most frequent therapy used followed by hormonal deprivation and radical prostatectomy. Independent factors to apply the treatment was the age $(\mathrm{OR}=5,6)$ and the risk groups $(\mathrm{OR}=2,8)$ in the year 2004 and only the age $(>70$ years vs $\leq 70$ ) in the year 1995 .

Conclusions: The evolution held in treatment therapies for prostate adenocarcinomas, made this disease in terms of treatment a multidisciplinary disease obtaining better results.

Keywords: Prostate cancer. Hormonotherapy. Quemotherapy.
} 
$\mathrm{E}$ 1 cáncer de próstata $(\mathrm{CP})$ representa el tumor urológico más frecuente en varones. Se ha descrito como la cuarta neoplasia en todo el mundo y la tercera en el varón, por detrás del melanoma y del cáncer de pulmón ${ }^{1}$. El cáncer de próstata que tiene una incidencia elevada en la población general, es una enfermedad que plantea muchos problemas clínicos. Además, el 20\% de los hombres sufrirán manifestaciones clínicas de esta enfermedad a lo largo de su vida ${ }^{2}$. Sin embargo, sólo el 20\% de los pacientes diagnosticados mueren por causa directa de la enfermedad y reduce la esperanza de vida de los afectados en unos 9 años ${ }^{2,3}$. Por ello, es importante conseguir una estadificación fiable para, en función de la estadificación y del pronóstico, proponer el tratamiento más adecuado y racional posible, en base a todo lo que se conoce, actualmente, sobre esta patología.

El enfoque al tratamiento está influido por la edad, las características del tumor y por los problemas médicos coexistentes. Los efectos secundarios de las diversas formas de tratamiento se deberán tener en cuenta al seleccionar el control apropiado. A pesar de la alta incidencia del cáncer de próstata, todavia existe controversia en cuanto al significado de los exámenes de detección, la evaluación más apropiada para su clasificación en diferentes estadios y el tratamiento óptimo para cada etapa de la enfermedad ${ }^{4}$. Un concepto importante que se debe tener en cuenta es el de cáncer latente, frente al cáncer clínico. En estudios necrópsicos se ha puesto de evidencia que el cáncer latente de próstata puede ser diagnosticado con uniformidad a partir de los 40 años en el 12\% de los varones, lo que supone una incidencia entre 10 y 15 veces mayor que el cáncer clínico. Actualmente, se desconoce el mecanismo por el que el cáncer de próstata latente se convierte en cáncer clínico.

El tratamiento definitivo se utiliza, generalmente, en hombres más jóvenes y sin enfermedades médicas concominantes graves, ya que tienen una mayor probabilidad de fallecer por este tumor que hombres de mayor edad o con enfermedades médicas graves concominantes. Aproximadamente, un $50 \%$ de los pacientes sufren una recidiva de la enfermedad después del tratamiento local consistente en radioterapia o cirugía radical $^{5-7}$. La tasa de supervivencia a los 5 años es del
85\% para los pacientes con cáncer de próstata que se diagnostican en una etapa precoz. En los últimos 30 años se ha conseguido diagnosticar el $70 \%$ de estos tumores precozmente. Generalmente, un porcentaje importante de los pacientes que tienen un cáncer de próstata localmente avanzado morirán por su tumor, aunque el promedio de supervivencia es de hasta 5 años. Si el tumor se ha propagado a órganos distantes, la supervivencia promedio es de 1-3 años y la mayoría de estos pacientes fallecerá por esta causa. Sin embargo, aún en este grupo de pacientes, se pueden observar cursos clínicos indoloros que se alargan muchos años ${ }^{8}$.

\section{MATERIAL Y MÉTODOS}

Realizamos un estudio retrospectivo sobre la base de datos de los pacientes diagnosticados de cáncer de próstata en el Servicio de Urología I del Hospital Universitario Central de Asturias (HUCA) en los años 1995 y 2004.

Se han incluido en nuestro estudio 203 pacientes de los 216 diagnosticados de adenocarcinoma de próstata en los dos años, 143 corresponden al año 2004 y 60 a 1995.

Se excluyeron del estudio los pacientes que:

- No tenían historia clínica completa.

- Tenían tumor de próstata no adenocarcinoma.

El estudio se basó en datos recogidos en la consulta externa, en su mayoría, evidenciándose una serie de diferencias y variables dependiendo del año en el que se recogieron, ya que en 1995 no se realizaban en el Servicio de Urología ecografias transrectales de próstata, lo que imposibilitaba la realización de biopsias ecodirigidas y la estadificación de un tumor visible en la ecografía como $\mathrm{T} 2$.

Se revisaron las historias clínicas de los pacientes que seleccionamos para el estudio recogiéndose los datos según un protocolo establecido previamente en el que se incluían las siguientes variables.

- Datos de filiación del paciente, que incluyen (nombre y apellidos, número de historia clínica, número de biopsia asignado en Anatomía Patológica, edad al diagnóstico y fecha de diagnóstico).

- Ecografia, tipo de ecografia, volumen prostático y alteraciones ecográficas. 
- Método de diagnóstico.

- Biopsias prostáticas previas al diagnóstico.

- Número de cilindros extraídos en la biopsia y número de cilindros positivos.

- Estadificación TNM.

- Pruebas de imagen complementarias (gammagrafía osea y escáner).

- Tratamiento realizado.

Respecto al tipo de ecografía realizada, ésta puede ser por vía abdominal (realizada por un radiólogo) o por vía transrectal (realizada por un urólogo) la cual consigue una medida más eficaz del volumen prostático y además se pudo constatar la presencia de varios tipos de alteraciones en la exploración, como la presencia de imágenes hipo o hiperecoicas, así como la alteración de la cápsula prostática o de las vesículas seminales. En cuanto al tratamiento realizado se incluyeron: Observación, Prostatectomía radical, Radioterapia externa, Braquiterapia u Hormonoterapia.

Los objetivos propuestos fueron los siguientes:

Determinar las pruebas complementarias empleadas en el diagnóstico, así como, las pautas de tratamiento empleadas en los tumores de próstata diagnosticados en los años 1995 y 2004.

Los resultados fueron almacenados en una base de datos y procesados mediante el programa SPSS versión 11 de aplicaciones estadísticas. Se estableció significación estadística para valores de p menores de 0,05 con intervalo de confianza del 95\%.

\section{RESULTADOS}

Volumen prostático

El volumen prostático se determinó por ecografía realizada por vía transrectal en 114 pacientes $(56,2 \%)$. Todas las determinaciones del volumen prostático realizadas en el año 1995 se llevaron a cabo mediante ecografía abdominal frente a un 15,4\% de las realizadas en el año 2004. El volumen medio observado fue de 43,7 $\pm 21,4$ (12-131) cc. El volumen medio determinado por ecografía abdominal $(52,35 \pm 23,55 \mathrm{cc})$ fue significativamente mayor $(\mathrm{p}<0,001)$ que el observado mediante ecografía transrectal $(38 \pm 17,7$ cc), lo que explicaría que los volúmenes observados en los tumores del año 1995 fueran superiores a los de la serie del año 2004.

\section{Biopsias}

Respecto a las biopsias y a los procedimientos diagnósticos se encontraron alteraciones en la ecografía transrectal, nódulos hipo o hiperecoicos, asimetría de vesículas seminales, rotura de cápsula prostática, entre otros, que sugerían neoplasia de próstata en 45 pacientes, todas ellas en el año 2004. En 48 pacientes se había realizado más de una biopsia, 8 de los cuales pertenecían a la serie del año 1995 y 40 al año 2004. A la mayoría de los pacientes se les había realizado una biopsia previa, a 1 paciente le habían realizado 4 biopsias, a 2 pacientes le realizaron 3 y en 10 casos tenían 2 biopsias anteriores a la biopsia diagnóstica de neoplasia. La mayoría de los pacientes correspondían a la serie del año 2004, excepto 2 pertenecientes a la serie de 1995 .

Se encontraron diferencias significativas $(\mathrm{p}<$ 0,001) en las medias del número de cilindros obtenidos en el año 1995 comparados con los del año 2004. La media de cilindros obtenidos en los pacien-

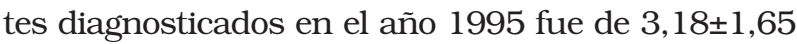
cilindros (1-6) y la del año 2004 de 6,1ะ0,97 cilindros (1-10). Se debe tener en cuenta que en 1995 sólo se realizaron 10 biopsias sextantes frente a casi la totalidad en el año 2004 (en 3 pacientes se recogieron menos de 6 cilindros y en 10 se obtuvieron más de 6 cilindros). No obstante, no se encontraron diferencias significativas en el número de cilindros positivos comparando las medias del año 1995 $(2,8 \pm 1,6)$ y del $2004(2,6 \pm 1,7)$. Sin embargo, el porcentaje medio de cilindros positivos, respecto al total de cilindros obtenidos, fue del 90,4\% en el año 1995 frente al 43,9\% en el año 2004.

\section{Tomografia Axial Computerizada}

Se realizó TC de estadificación en el 61,7\% de los tumores diagnosticados en el año 1995 frente al 49,7\% de los diagnosticados en el año 2004. En ninguno de los años se encontró asociación significativa de la realización del TC con la suma de Gleason ni con la concentración del PSA, aunque fue mayor la frecuencia de realización de escáner cuando ambos parámetros estaban elevados. Se realizó un TC en el 41,1\% de los pacientes con una concentración de PSA menor de $10 \mathrm{ng} / \mathrm{ml}$, correspondiendo un $13,9 \%$ a la serie de pacientes del año 1995 frente a un $54,9 \%$ en los pacientes del año 2004. No se encontró asociación estadística entre la realiza- 
ción de un TC y el tacto rectal en la serie del año 1995 pero sí en la de 2004 ( $\mathrm{p}=0,021)$, de manera que cuando el tacto rectal fue sospechoso se realizaron más frecuentemente TAC en los pacientes diagnosticados este año.

\section{Gammagrafia Ósea}

Globalmente se realizó una gammagrafia ósea al $69,7 \%$ de los pacientes, que se distribuyen en un $80 \%$ y un $65,2 \%$ de los casos diagnosticados en los años 1995 y 2004, respectivamente. Se observó una asociación significativa $(p=0,026)$ en la frecuencia de realización de gammagrafia ósea entre la serie de pacientes de los años 1995 y 2004, mayor de la esperada. Igualmente se encontró una asociación significativa $(\mathrm{p}=0,005)$ entre la positividad de la gammagrafia y el porcentaje de pacientes con metástasis óseas en la serie de pacientes del año 1995, encontrándose 14 casos con metástasis óseas $(23,3 \%)$, de los que 10 pacientes $(71,4 \%)$ presentaron focos múltiples. En el año 2004, se diagnosticaron metástasis óseas en 9 casos (6,3\%), habiendo en 8 de ellos metástasis diseminadas $(88,9 \%)$. Hay que decir que aunque existieron diferencias significativas en las concentraciones de PSA $(\mathrm{p}<0,0019)$ entre los pacientes que tuvieron metástasis óseas y aquellos que no las presentaron, sin embargo, no se encontraron dichas diferencias entre los pacientes en los que se realizó gammagrafía ósea y aquellos en los que no se solicitó, considerando globalmente las dos series de pacientes. De hecho, se realizó gammagrafia ósea en 59 casos (29\% del total de pacientes) con un valor de PSA menor de $10 \mathrm{ng} / \mathrm{ml}, 9$ pertenecían al año 1995 (15\%) y 50 al 2004 (34,9\%). Se encontró una asociación significativa del hallazgo de metástasis óseas en gammagrafia ósea solicitada por un tacto rectal sospechoso $(\mathrm{p}=0,01)$ por un lado, y con una suma de Gleason mayor de $7(\mathrm{p}<0,001)$ por otro, considerando la serie de pacientes de los dos años conjuntamente. Sin embargo, no se evidenciaron tales asociaciones al analizar la realización de dicha prueba y el resultado del tacto o del grado de la biopsia.

\section{TRATAMIENTO}

Encontramos diferencias significativas $(p<0,001)$ en la terapia empleada según el año de diagnóstico. Los pacientes correspondientes al año 1995 se trataron fundamentalmente con deprivación androgénica, seguido por prostatectomía radical y ocasionalmente, con radioterapia externa. En los pacientes diagnosticados en el año 2004 el tratamiento más empleado fue la radioterapia (externa y/o braquiterapia), seguido de la hormonoterapia y la prostatectomía radical, permaneciendo el $4,2 \%$ de pacientes en programas de observación ( $\mathrm{n}=6$ ) (Fig. 1).

Globalmente se encontraron diferencias significativas en la media de edad según el tipo de tratamiento empleado $(\mathrm{p}<0,001)$, de manera que los pacientes tratados con hormonoterapia o sometidos a observación presentaban edades medias más elevadas que el resto. Además, entre los casos tratados de forma radical se observó que la radioterapia fue aplicada en pacientes con más edad que los que fueron intervenidos (prostatectomía radical). Al analizar los grupos de edad según el año de diagnóstico, se observó igualmente una asociación significativa $\left(\mathrm{Chi}^{2}\right)$ de la terapia radical con la edad, tendiendo a la hormonoterapia en edades más avanzadas y al tratamiento radical en pacientes más jóvenes, si bien no se empleó observación ni obviamente braquiterapia en los pacientes diagnosticados en el año 1995 (Tabla 1, Fig. 2 y 3).

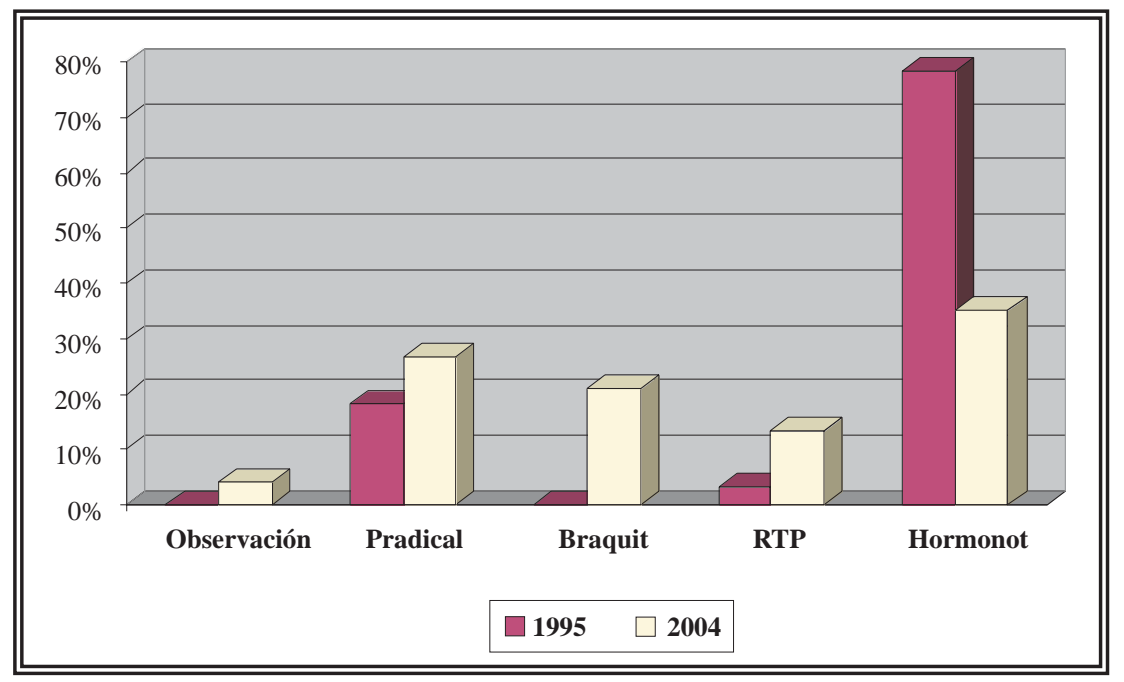

FIGURA 1. Porcentaje de pacientes según el tratamiento realizado. (Pradical: Prostatectomía radical. Braquit: Braquiterapia. RTP: Radioterapia externa. Hormonot: Hormonoterapia. 
Tabla 1

Tratamiento según grupos de edad. (Entre paréntesis, porcentaje de casos para cada tratamiento)

\begin{tabular}{lcccccc}
\hline Año & Tratamiento & $\begin{array}{c}\mathbf{5 0 - 5 9} \text { años } \\
\mathbf{n}(\%)\end{array}$ & $\begin{array}{c}\mathbf{6 0 - 6 9} \text { años } \\
\mathbf{n}(\%)\end{array}$ & $\begin{array}{c}\mathbf{7 0 - 7 5} \text { años } \\
\mathbf{n}(\%)\end{array}$ & $\begin{array}{c}>75 \text { años } \\
\mathbf{n}(\%)\end{array}$ & $\begin{array}{c}\text { Total } \\
\mathbf{n}(\%)\end{array}$ \\
\hline 1995 & Pradical & $4(36,4)$ & $6(54,5)$ & $1(9,1)$ & $0(0)$ & $11(100)$ \\
& Radiot ext & $0(0)$ & $1(50,0)$ & $1(50,0)$ & $0(0)$ & $2(100)$ \\
& Hormonot & $3(6,4)$ & $4(8,5)$ & $16(34,0)$ & $24(51,1)$ & $47(100)$ \\
Total & & $7(11,7)$ & $11(18,3)$ & $18(34,0)$ & $24(40,0)$ & $60(100)$ \\
2004 & Observación & $0(0)$ & $1(16,7)$ & $1(16,7)$ & $4(66,7)$ & $6(100)$ \\
& Pradical & $8(21,1)$ & $25(65,8)$ & $4(10,5)$ & $1(2,6)$ & $38(100)$ \\
& Braquiterapia & $3(10,0)$ & $13(43,3)$ & $14(46,7)$ & $0(0)$ & $30(100)$ \\
& Radiot ext & $1(5,3)$ & $9(47,4)$ & $7(36,8)$ & $2(10,5)$ & $19(100)$ \\
& Hormonot & $3(6,0)$ & $10(20,0)$ & $19(38,0)$ & $18(36,0)$ & $50(100)$ \\
& & $15(10,5)$ & $58(40,6)$ & $45(31,5)$ & $25(17,5)$ & $143(100)$ \\
\hline
\end{tabular}

Hormonol: Hormonoterapia. Pradical: Prostatectomía radical. Radiol ext: Radioterapia externa.

Evidenciamos una asociación significativa entre la existencia de metástasis y el empleo de hormonoterapia tanto en el año 1995 ( $\mathrm{p}=0,019)$ como en el año 2004 ( $\mathrm{p}<$ $0,0019)$. No obstante, también se empleó deprivación androgénica en 32 y 39 pacientes que no presentaban evidencia clínica de metástasis en los años 1995 y 2004, respectivamente. En este grupo el 93,8\% de los pacientes tratados en el año 1995 eran mayores de 70 años frente al $74,3 \%$ de los tratados en el año 2004. Además, tanto en la serie de pacientes del año 1995 como en la del año 2004, los menores de 70 años se sometieron a deprivación androgénica debido a la existencia de morbilidad asociada importante $(n=48)$ y/o presencia de tumores localmente avanzados

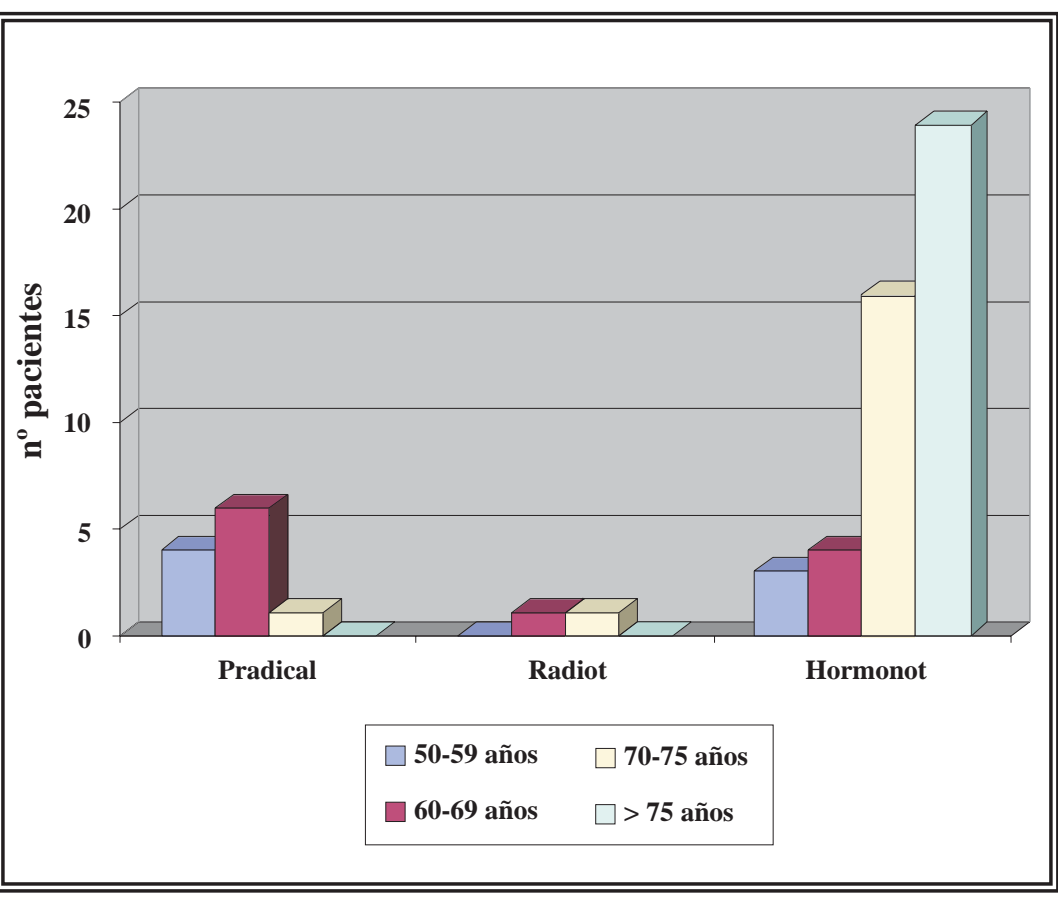

FIGURA 2. Tratamiento en función del grupo de edad en los pacientes diag nosticados en el año 1995.
T3-4 (3 casos localmente avanza-

dos en el año 1995 y 7 casos en el año 2004), y/o suma de Gleason mayor de $6(n=30)$ y/o concentraciones elevadas de PSA. Ninguno de los 6 casos a los que se aplicó observación (todos en el año 2004) presentaron metástasis clínicas. Dos pacientes del año 2004 fueron sometidos a tratamiento radical, bien prostatectomía $(n=1)$ o radioterapia externa $(n=1)$, a pesar de tener evidencia clínica de metástasis ganglionares. En cuanto a los tumores localizados, no existió diferencia significativa entre la terapia empleada y los grupos de riesgo en la serie de pacientes del año 1995, observándose, sin embargo, una diferencia significativa en los pacientes tratados en el año $2004(\mathrm{p}<0,001)$. De esta manera, la observación, prostatectomía radical o braquiterapia se emplearon fundamentalmente en tumores de bajo riesgo y la radioterapia externa y hormonoterapia en los de medio y alto riesgo (Tabla 2). 


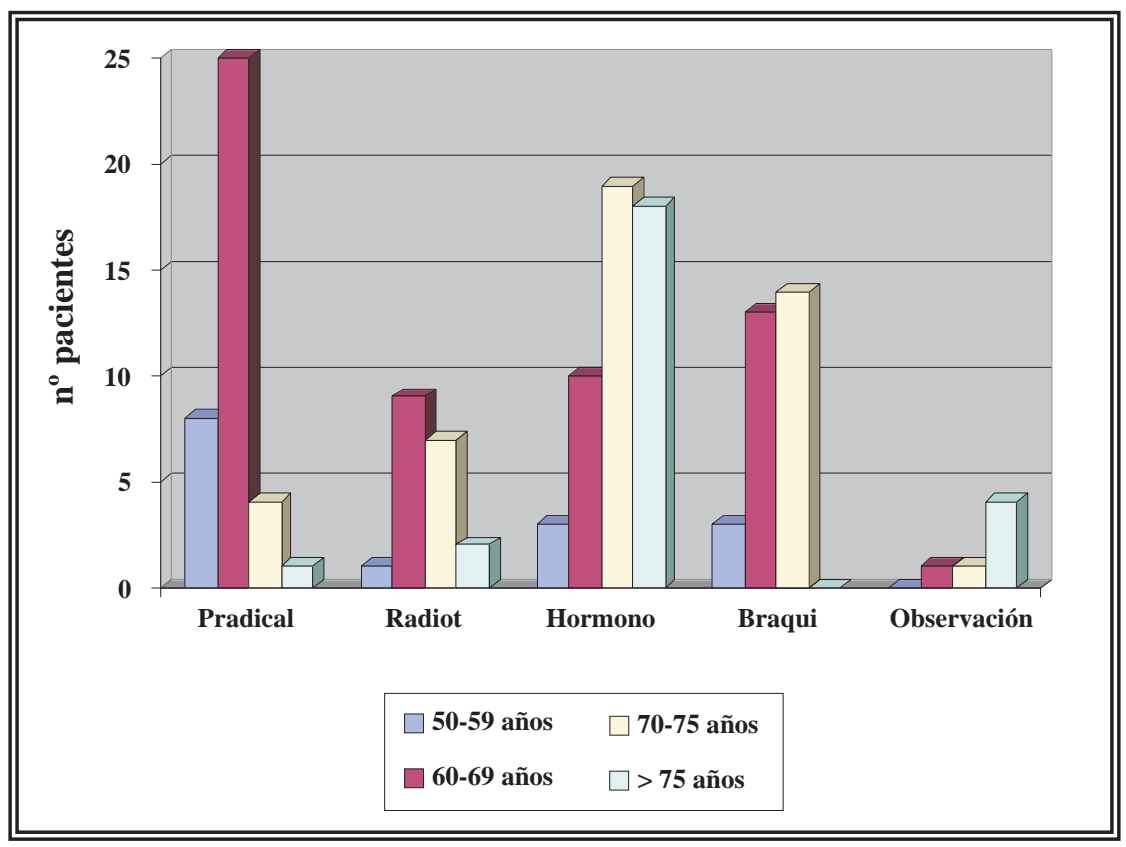

FIGURA 3. Tratamiento en función del grupo de edad en los pacientes diagnosticados en 2004. (Braqui: Braquiterapia. Hormona: Hormonoterapia. Pradical: Prostatectomia radical. Radiol: Radioterapia externa).

Tabla 2

Tratamiento en pacientes diagnosticados en el año 2004 en función de los grupos de riesgo

\begin{tabular}{|c|c|c|c|c|c|}
\hline \multirow[t]{2}{*}{ Año } & \multirow[t]{2}{*}{ Tratamiento } & \multicolumn{3}{|c|}{ Grupos de riesgo } & \multirow[b]{2}{*}{$\begin{array}{l}\text { Total } \\
\text { n (\%) }\end{array}$} \\
\hline & & $\begin{array}{c}\text { Bajo } \\
\text { n (\%) }\end{array}$ & $\begin{array}{c}\text { Medio } \\
\text { n (\%) }\end{array}$ & $\begin{array}{l}\text { Alto } \\
\text { n (\%) }\end{array}$ & \\
\hline \multirow[t]{5}{*}{2004} & Observación & $5(83,3)$ & $0(0)$ & $1(16,7)$ & $6(100)$ \\
\hline & Pradical & $23(62,2)$ & $12(32,4)$ & $2(5,4)$ & $37(100)$ \\
\hline & Braquiterapia & $17(56,7)$ & $13(43,3)$ & $0(0)$ & $30(100)$ \\
\hline & Rtp externa & $1(5,6)$ & $5(27,8)$ & $12(66,7)$ & $18(100)$ \\
\hline & Hormonoterapia & $12(27,9)$ & $13(30,2)$ & $18(41,9)$ & $43(100)$ \\
\hline Total & & $58(43,3)$ & $43(32,1)$ & $33(24,6)$ & $134(100)$ \\
\hline
\end{tabular}

En el estudio de regresión logística (tipo Wald), los factores que resultaron ser independientes del tratamiento aplicado, radical o mediante deprivación hormonal, en los pacientes con tumores localizados y diagnosticados en el año 2004, son los siguientes: la edad mayor o menor de 70 años ( $<<0,001$ ) con una odds ratio de 5,6 (IC del 95\%: $2,35-13,28$ ) y el grupo de riesgo (alto frente a mediobajo) ( $\mathrm{p}=0,029$ ), con una OR de 2,8 (IC 95\%: 1,17,2 ), quedando excluida la existencia de morbilidad asociada. Cuando se realiza este análisis sobre la serie de pacientes del año 1995, el único factor independiente $(\mathrm{p}<0,001)$ ha sido la edad (mayor o menor de 70 años).

\section{DISCUSIÓN}

El presente estudio, continuación de uno previo en el que se evaluaron las características clínicas y anátomo-patológicas de los cánceres de próstata diagnosticados en Oviedo en los años 1995 y 2004, fue diseñado para evaluar y comparar el patrón de diagnóstico así como el tratamiento empleado en los tumores de próstata diagnosticados en el Servicio de Urología 1 del HUCA en esos mismos años.

Se observó, en las dos series de nuestro estudio, una asociación significativa entre el estadio local y la presencia de metástasis de tal manera que los tumores con estadio T3 o T4 se asociaron, más frecuentemente que el resto, a la presencia de metástasis ganglionares y óseas. Otro dato interesante fue la presencia de metástasis óseas en el momento del diagnóstico. Se detectó una asociación significativa entre la positividad de la gammagrafia ósea y el año de diagnóstico, ya que en el año 1995 un $23,3 \%$ de los pacientes presentaban metástasis óseas frente a sólo el 6,9\% de los pacientes en la serie del año 2004. Estos resultados son similares a los publicados por Murphy et al. ${ }^{9}$, que encontraron un $21 \%$ de pacientes con $\mathrm{CP}$ y metástasis óseas, entre 1981 y 1990, frente al 10\% de los revisados en el año 1995. El hecho de esta anticipación en el tiempo respecto a nuestra serie, puede ser debido a que en EEUU se comenzó a usar de forma habitual el PSA antes que en España. En la actualidad en EEUU se realiza cribado del cáncer de próstata, utilizando la determinación del PSA.

Respecto a la variable del tratamiento de los pacientes diagnosticados de cáncer de próstata en nuestra serie, se encontró una asociación significativa entre la terapia empleada y el año de diagnóstico. En el año 1995 el tratamiento más frecuentemente empleado fue la hormonoterapia o deprivación androgénica (78,3\% de los casos), un $18,4 \%$ fueron tratados con cirugia radical y un $3,3 \%$ con 
radioterapia, mientras que en el año 2004 los tratamientos más empleados fueron la hormonoterapia, la radioterapia (incluyendo radioterapia externa y la braquiterapia) y la prostatectomía radical en un $35 \%, 34,3 \%$ y $26,6 \%$ de los casos, respectivamente. Esta diferencia significativa entre los diferentes tratamientos en los años 1995 y 2004 está relacionada con el estadio tumoral, la edad y la oferta terapéutica en el momento del diagnóstico. En el año 1995 la edad media en el momento del diagnóstico era más alta que en el año 2004, había un mayor porcentaje de pacientes con metástasis al diagnóstico y, entonces, no se ofertaba la braquiterapia. Herraz-Amo et al. ${ }^{10}$ en su revisión de los cánceres de próstata diagnosticados en la Comunidad de Madrid en el año 2000, observaron que el tratamiento más utilizado también fue la hormonoterapia (35\% de los casos), seguido de la prostatectomía radical (34\%) y de la radioterapia (25\%).

Globalmente encontramos también diferencias significativas entre la edad en el momento del diagnóstico y el tratamiento empleado, de tal manera que los pacientes tratados con hormonoterapia y observación presentaban edades medias más altas que el resto. Esto se correlaciona con otros resultados obtenidos en nuestro estudio, por ejemplo, los pacientes con edad media más alta tenían unas concentraciones medias de PSA más elevadas, y score de Gleason mayor. Estos resultados coinciden con otros descritos en la bibliografia ${ }^{9-11}$.

En lo referente a los factores que resultaron independientes para explicar que los pacientes con tumores localizados fueran tratados de forma radical o mediante deprivación hormonal, en la serie de pacientes del año 2004, se observó que tanto la edad (mayor o menor de 70 años) y el grupo de riesgo (alto frente a medio o bajo) se comportaron como tal, excluyéndose la existencia de morbilidad asociada, mientras que en el año 1995 únicamente la edad (mayor o menor de 70 años) resultó ser factor independiente, quedando fuera el grupo de riesgo del paciente y la presencia de morbilidad asociada.

Finalmente, vistos los resultados obtenidos en nuestro estudio y coincidentes con otros llevados a cabo en otras comunidades respecto a la evolución en el diagnóstico, pronóstico y tratamiento del cáncer de próstata en la última década, podemos concluir que la forma de presentación del cáncer de próstata en nuestra comunidad ha cambiado, ya que el uso generalizado de un marcador próstatoespecífico como es el PSA, ha permitido diagnosticar más tumores, en estadios más precoces y en pacientes más jóvenes. Sin embargo, no es lo suficientemente específico de cáncer de próstata y obliga en ocasiones a la realización de muchas biopsias para obtener un diagnóstico de la enfermedad, si bien con el uso de los nuevos parámetros del PSA, como el índice, la velocidad o la densidad del PSA se ha conseguido disminuir un número importante de biopsias innecesarias. Por otra parte, se han aumentado los tratamientos radicales con intención curativa en los pacientes más jóvenes con menor morbilidad y con tumores menos agresivos. La evolución experimentada en las distintas técnicas terapéuticas ha convertido al cáncer de próstata en una enfermedad multidisciplinar obteniendo una gran mejoría en los resultados del tratamiento. Pese a todo esto, el desconocimiento existente en la actualidad acerca de la historia natural del cáncer de próstata y de su evolución en la mayoría de los casos, hace que sea necesario la realización de grandes estudios prospectivos, los cuales se están llevando a cabo, para determinar si el cribado del cáncer de próstata mediante el uso del PSA puede ser útil o no.

\section{RFERENCIAS}

1. Parkin DM, Pissani P, Ferlay J. Estimates of the worldwide incidence of eighteen mayor cancers. Int J Cancer. 1993 Jun 19;54(4):594-606.

2. Nesbit RM, Plumb RT. Prostatic carcinoma: a follow up on 795 patients treated prior to the endocrine era and a comparison of survival rates between these and patients treated by endocrine therapy. Surgery 1996;20 (3):263

3. Wingo PA, Landis S, Ries LAG. An adjustment to the 1997 estimate of new prostate cancer cases. Cancer. 1997 Nov 1;80(9):1810 242.

4. Garnick MB. Prostate cancer: screening, diagnosis and management. Ann Intern Med. 1993 May 15;118(10):804-818.

5. Zincke H, Bergstralh EJ, Blute ML, Myers RP, Barrett DM, Lieber MM. Radical prostatectomy for clinically localized prostate cancer: longterm results of 1143 patients from a single institution. J Clin Oncol. 1994 Nov; 12(11):2254-2263.

6. Zincke H, Oesterling JE, Blute ML, Bergstralh EJ, Myers RP, Barrett DM. Long-term (15 years) results after radical prostatectomy for clinically localized (stage T2c or lower) prostate cancer. J Urol 1994;152(7): 1850-1857.

7. Goodin S, Rao KV, DiPaola RS. State-of-the-art treatment of metastatic hormone-refractary prostate cancer. Oncologist. 2002;7(4):360-370.

8. Gittes RF. Carcinoma of the prostate. N Eng J Med 1991;324(9):236-245

9. Murphy M, Johnston C, Whelan P, Rider L, LLoyd SN. Changing trends in prostatic cancer. BJU International 1999;83(4):786-791.

10. Herranz Amo F, Arias Funez F, Arrizabalaga Moreno M, Calahorra Fernández FJ, Carballido Rodríguez J, Diz Rodríguez R, et al. El cáncer de próstata en la Comunidad de Madrid en el año 2000. IIIEstudio de extensión tumoral. Act Urol Esp 2003;27(8):411-417.

11. Mettlin CJ, Murphy GP. Why is prostate cancer rate declining in the United States?. Cancer 1998;82(4):229-245.

Dr. M. Álvarez-Múgica

Email: malvarez79@mixmail.com

(Trabajo recibido el 31 de julio de 2006) 